\title{
Preface
}

\section{Overview of the Special Issue from the Fifth International Conference on Establishment Surveys (ICES-V)}

\author{
Katherine J. Thompson ${ }^{1}$, Polly Phipps ${ }^{2}$, Darcy Miller ${ }^{3}$, and Ger Snijkers ${ }^{4}$
}

\section{Establishment Surveys. What Are They and Why Dedicate an Entire Conference Series to Them?}

The Encyclopedia of Survey Research Methods provides the following definition:

"An establishment survey is a survey that seeks to measure the behavior, structure, or output of organizations rather than individuals. Establishment surveys include surveys of business that are critical to our understanding of trends in the economy." Establishment surveys provide economic measures that change economies. They are inputs to National Accounts, to Price Indices, and to Gross Domestic Products. Economic indicators move markets. Interest rate hikes and decreases follow the release of economic statistics. Definitions change as economies mature or become global. Data users include policymakers, business communities, and economists. The demand for timely, relevant, and accurate economic measures continues to increase, even as establishment surveys are plagued by the same decreasing response rates and budget challenges as their household counterparts.

The formal definition of an establishment survey, while accurate, is not entirely enlightening. Household surveys are more intuitive: the survey unit is a person or group of persons, the intent is to measure some form of human characteristics, behavior, and/or opinions (e.g., employment, victimization experience, political preferences, expenditures, etc.), and the questionnaire recipient is often best equipped to answer the questions. A large portion of the survey sampling and survey methodology literature focuses on household surveys. Multi-stage designs are commonplace, selecting households within

${ }^{1}$ Guest Editor. U.S. Census Bureau, Economic Statistical Methods Division, 4600 Silver Hill Road, Washington, DC 20233, U.S.A. Email: Katherine.j.thompson@census.gov

${ }^{2}$ Guest Editor. Bureau of Labor Statistics, Office of Survey Methods Research, Suite 5930, 2 Massachusetts Ave N.E., Washington, D.C. 20212. Email: phipps.polly@bls.gov

${ }^{3}$ Guest Associate Editor. National Agricultural Statistics Service, Research and Development Division, 1400 Independence Avenue SW, Washington, DC, U.S.A. Email: Darcy.Miller@nass.usda.gov

${ }^{4}$ Guest Associate Editor. Statistics Netherlands, Methodology Department, CBS-weg 11, 6412 EX Heerlen, Netherlands. Email: g.snijkers@cbs.nl

Acknowledgments: First, we thank the JOS Editorial Board for allowing us to publish this special issue. We are grateful for the forum. Second, we thank all of the authors for their interesting submissions and for their patience and perseverance with revisions. Last, we thank the referees who generously donated their time and provided useful and timely reviews. This allowed us to produce a varied volume with no delays and greatly simplified our job. 
geographic areas. In National Statistical Institutes (NSI), care is taken to minimize overlap with other household surveys, so that respondents are not burdened with participation in more than one survey at a time. Bayesian methodologists employ multivariate normal distributions in their models. Cognitive psychologists use established procedures to design, test, and assess survey questions and questionnaires. The human aspect of collection is emphasized in interviewer training, and proxy responses can be acceptable. Since the collection units have the same definitions, other source data - such as administrative data or social media data - can be directly integrated into the survey estimation procedure.

Establishment surveys are different. The survey unit could be a business (establishment, company, firm), farm, institution (school, government) - basically, anything other than a household or a person. Within the same survey, the reporting unit can be the unit sampled from the frame or another entity entirely, such as an accounting office (or offices) within a firm or the distribution center for a warehouse. Reporting units can provide consolidated information for a set of sampling units, like the state government providing pension data for government offices in sampled counties. In contrast with household surveys, establishment survey populations are generally skewed, with the largest cases providing the majority of data for the estimated totals. Stratified one-stage sample designs are frequently used, with the largest units sampled with probability equal to one (certainty units), and these certainty units are often included in more than one survey. Model misspecification is commonplace, as normal theory approximations rarely hold. Unit classification into a single category such as industry may not be possible; for example, a large company may operate in several different sectors. Definitions used in official statistics may hinder frame development for hard-to-reach populations, as discussed in the Young et al. article in this issue.

Collection challenges are entirely different for establishment surveys. Large companies and governments may assign a single questionnaire to different departments, divisions or regions, in order to produce a consolidated response. Establishment surveys have a different cognitive model, with records playing an important role. Because of their important contribution to survey totals, larger businesses are treated differently. Research on collection methods and contact strategies has been largely focused on obtaining accurate reported data from large businesses. Indeed, many NSIs have dedicated account manager offices, whose primary purpose is to establish and maintain ongoing contact and support with the largest sampled units as described in this issue's article by Giesen and colleagues. Others are working towards data sharing and integration as discussed by Buiten et al. in this issue. McCarthy and colleagues proposed holistic "Bento Box" approach to questionnaire testing discussed in this issue stems directly from the collective set of collection challenges posed by skewed populations, specialized terminology, multiple respondents, and record extraction. As opposed to household surveys, experimentation in establishment surveys is scarce, rendering especially valuable the contribution in this issue by the Tuttle et al. article that describes an experiment aimed at optimizing the contact strategy.

To complicate things further, the survey unit may not remain constant. A business could change in composition over time due to mergers, acquisitions, or divestitures, a farm could grow new crops, or a local school building could become an administration building. The 
same survey unit could be classified entirely differently after data collection. "Stratum jumpers" (units that become much lower or smaller in size between sample selection and implementation) distort estimates and variance estimates. Outlier detection and treatment procedures that alleviate these distortions as discussed in the Mulry et al. article in this issue are therefore extremely useful.

And of course, there is a well-known "unit problem" within the establishment survey community complicating everything. For instance, there is no guaranteed correspondence between the statistical unit used for sampling and the administrative unit established for tax collection, as is discussed by Buiten et al. Other source data such as transaction data may not match to the survey data because of unit differences, but may have severe coverage issues, as discussed in the Di Cecco et al. article presented in this issue.

So not only are establishment surveys different from household surveys, they are deceptively difficult to administer. Indeed, response rate and potential bias are discussed in several articles in this issue, including those by Beck et al., Kaputa and Thompson, and Earp et al. NSIs are being challenged to produce more detailed estimates with quicker turnaround and respond to these challenges creatively, as demonstrated in the Fröhlich article and the two small area estimation articles by Zimmerman and Munnich and by Luzi et al.

In 1993, methodologists met in Buffalo, New York to launch a series of conferences that brought together an international community interested in sharing research, discussing issues, and developing a community based entirely around establishment surveys and censuses. As this community solidified, the International Conference on Establishment Surveys (ICES) has progressed from a seven-year conference cycle to a four-year conference cycle. The conference participation has evolved as the programs have seasoned, and participation encompasses official statisticians, private sector methodologists, and academics who come to share experiences, learn from others, or simply to learn about establishment surveys. This growing international community is evidenced by several articles in this issue with co-authors from a variety of different statistical agencies. It is further evidenced by this issue's independently submitted "Letter to the Editor," which specifically cites the presentations and sessions at the ICES-V conference as (partial) motivation for the subsequent creation of the Intersecretariat Working Group on National Accounts (ISWGNA) Task Force on Statistical Accounts by the United Nation Statistics Division.

The common theme throughout the conference series is the collection of data from nonhousehold populations, with specific topics evolving over time as expertise has increased, technology has improved, and alternative data sources have emerged. The program has likewise matured, now featuring introductory overview lectures on important common topics, such as questionnaire design, classification, adaptive design, and disclosure avoidance, short courses, and a varied suite of invited, topic contributed, and contributed paper presentations. Keynote speakers bracket the program. The latest conference featured a student contest, with numerous entrants and the presentation of three research papers as an outcome (two winners and an honorable mention). As this issue is released, planning for the Sixth ICES conference is well underway.

With this issue, we hope to strengthen the commitment to this community for those who are already active participants. Past and present ICES attendees will recall the breadth of 
topics discussed and the level of detail, problem solving, and collaboration. This issue highlights a sampling of the interesting and varied challenges posed by establishment surveys (as discussed above) and illustrates the ingenuity of the vibrant community dedicated to administering them. More information about ICES-V is available at www.amstat.org/ASA/Meetings/ICES. If you haven't been familiar with either, we hope that this issue inspires you in this direction, perhaps even to the ICES-VI conference scheduled for June 2020 in New Orleans.

\section{In this Issue}

This issue presents a set of peer-reviewed articles based on papers presented at the Fifth International Conference on Establishment Surveys (ICES-V), held in Geneva, Switzerland in June 2016. We begin with the keynote speech by Peter van de Ven, Head of National Accounts, Statistics Directorate in the Organisation for Economic Co-operation and Development entitled "Economic Statistics: How to Become Lean and Mean." Mr. van de Ven's article opens the issue (and the conference) by reminding us of the importance of producing accurate and relevant economic measures.

The remaining articles are grouped into three themes, with some unavoidable overlap in selected cases: (1) Getting the Data, (2) Assessing and Treating the Data, and (3) Integrating Survey Data with Other Sources.

\subsection{Getting the Data}

Young, L. "Exploring a Big Data Approach to Building a List Frame for Urban Agriculture: A Pilot Study in the City of Baltimore." This article explores using alternative data sources such as websites to build a sampling frame for a hard-to-enumerate population.

McCarthy, J.S., Ott, K., Ridolfo, H., McGovern, P., Sirkis, R. and Moore, D. "Combining Multiple Questionnaire Testing Methods: Planning and Considerations for a Bento Box Approach to Testing for the 2017 Census of Agriculture." The authors present a case study in developing an effective questionnaire using a standardized system that addresses issues unique to establishment surveys.

Tuttle, A.D., Beck, J.S., Willimack, D.K., Hernandez, A., Tolliver, K.P, and Fan, C. "Experimenting With Contact Strategies in Business Surveys." This article presents the results of experimental testing of alternative mailing strategies to achieve timely and costeffective survey response.

Giesen, D., Vella, M., Brady, Jr., C.F., Brown, P., Ravindra, D., Vaasen-Otten, A. "Establishing and Maintaining a Relationship with Businesses: a Comparison of Response Burden Management in Official Business Surveys at the U.S. Census Bureau, Statistics Netherlands, Statistics Canada and Statistics New Zealand." The authors present theory on and practices for measuring and monitoring response burden, as well as the main burden reduction strategies used in four countries.

Buiten, G., Snijkers, G., Saraiva, P., Erikson, J., Erikson, A-G., and Born, A. "Business Data Collection: toward Electronic Data Interchange. Experiences in Portugal, Canada, Sweden and the Netherlands with EDI." The authors delineate data quality and technological challenges in four countries using electronic system-to-system data capture, 
where data is transferred directly from businesses to governmental organizations, like the tax office and statistical agency.

Kaputa, S.J. and Thompson, K.J. "Adaptive Design Strategies for Nonresponse FollowUp in Economic Surveys." This article describes optimal allocation methods for subsampling nonrespondents with simultaneous objectives of allocating high proportions of sample in domains that indicate potential nonresponse bias (based on quantitative metrics) and of equalizing response rates across all domains.

\subsection{Assessing and Treating the Data}

Earp, M., Toth, D., and Phipps, P. "Assessing Nonresponse in a Longitudinal Establishment Survey." The authors apply regression tree methods to a monthly panel survey to identify establishment characteristics associated with nonresponse during frame refinement, enrollment, and data collection over time, using the results to model nonresponse bias.

Mulry, M., Kaputa, S., and Thompson, K.J. "Setting M-estimation Parameters for Detection and Treatment of Influential Values." This article tackles the practical problem of implementing an outlier detection and treatment method in an ongoing survey using a highly parameterized statistical method.

Fröhlich, M. "Nowcasting Austrian Short Term Statistics." This article presents research into methods of generating reliable preliminary short-term statistics estimates produced under a reduced timeframe using highly sophisticated multivariate time series models.

\subsection{Integrating Survey Data with Other Sources}

Zimmermann, T. and Munnich, R. "Small Area Estimation with A Lognormal Mixed Model under Informative Sampling." The authors employ lognormal mixture models to approximate relationships between variables of interest and auxiliary variables from skewed business data to produce small area estimates. They extend the empirical best prediction (EBP) approach to compensate for informative sampling in these situations, providing diagnostic measures and an empirical assessment.

Luzi, O., Solari, F., and Rocci. F. "A Study of Small Area Estimation for Italian Structural Business Statistics." This article provides an empirical evaluation of different auxiliary data sources and small area estimators to produce detailed estimates that cannot be estimated directly.

Di Cecco, D., Di Zio, M., Filipponi, D., and Rocchetti, I. "Population Size Estimation Using Incomplete Lists With Overcoverage." This article presents a method for developing a single population estimate using varied sources of administrative or other source data, each with their own coverage requirements and errors.

\section{Reference}

Encyclopedia of Survey Research Methods (2008, published online 2011), edited by P.J. Lavrakas. Doi: http://dx.doi.org/10.4135/9781412963947. 